\title{
Regularity, depth and arithmetic rank of bipartite edge ideals
}

\author{
Manoj Kummini
}

Received: 14 October 2008 / Accepted: 11 February 2009 / Published online: 27 February 2009

(C) Springer Science+Business Media, LLC 2009

\begin{abstract}
We study minimal free resolutions of edge ideals of bipartite graphs. We associate a directed graph to a bipartite graph whose edge ideal is unmixed, and give expressions for the regularity and the depth of the edge ideal in terms of invariants of the directed graph. For some classes of unmixed edge ideals, we show that the arithmetic rank of the ideal equals projective dimension of its quotient.
\end{abstract}

Keywords Monomial ideals · Graded free resolutions · Arithmetic rank

\section{Introduction}

Let $G$ be a simple graph on a finite vertex set $V$ without any isolated vertices. Let $\mathbb{k}$ be a field. Set $R=\mathbb{k}[V]$, treating the elements of $V$ as indeterminates. Let $I$ be the edge ideal of $G$ in $R$, i.e., the ideal generated by the square-free quadratic monomials $x y$, where $x, y \in V$ and there is an edge between $x$ and $y$ in $G$. In this paper, we study (Castelnuovo-Mumford) regularity, depth and arithmetic rank of edge ideals of bipartite graphs. Recall that $G$ is said to be bipartite if there exists a partition $V=V_{1} \bigsqcup V_{2}$ such that every edge in $G$ is of the form $x y$ with $x \in V_{1}$ and $y \in V_{2}$.

When $I$ is unmixed (more generally, when $G$ has a perfect matching - see Section 2 for details), we have that $\left|V_{1}\right|=\left|V_{2}\right|=$ ht $I$. To such a bipartite graph, we associate a directed graph $\mathfrak{d}_{G}$ on the vertex set $\{1, \ldots$, ht $I\}$. This is motivated by a paper of J. Herzog and T. Hibi [4] which studies a similar association between posets and bipartite graphs with Cohen-Macaulay edge ideals. Using this, we show that

Theorem 1.1 Let $G$ be an unmixed bipartite graph with edge ideal I. Then $\operatorname{reg} R / I=\max \left\{|A|: A\right.$ is an antichain in $\left.\mathfrak{d}_{G}\right\}$. In particular, reg $R / I$ is the maximum size of a pairwise disconnected set of edges in $G$.

M. Kummini $(\bowtie)$

Purdue University, Lafayette, IN 47907, USA

e-mail: nkummini@math.purdue.edu 
We say that $G$ is unmixed (respectively, Cohen-Macaulay) if $R / I$ is unmixed (respectively, Cohen-Macaulay). The notion of pairwise disconnected sets of edges in graphs was introduced by X. Zheng [19] who showed that if $I$ is the edge ideal of a tree (an acyclic graph) then reg $R / I$ is the maximum size of a pairwise disconnected set of edges [19, Theorem 2.18]. Additionally, see [5, Corollary 6.9], for the same conclusion for the edge ideals of chordal graphs. For arbitrary graphs, the maximum size of a pairwise disconnected set of edges is a lower bound for $\operatorname{reg} R / I$; this follows essentially from [6, Lemma 2.2].

A strong component of a directed graph is a set of vertices maximal with the property that for every $i, j$ in the set, there is a directed path from $i$ to $j$. The following statement about depth, which follows from Corollary 3.7, has also been observed by C. Huneke and M. Katzman:

Theorem 1.2 Let $G$ be an unmixed bipartite graph, with edge ideal I and associated directed graph $\mathfrak{d}_{G}$. If $\mathfrak{d}_{G}$ has $t$ strong components, then depth $R / I \geq t$.

The problem of determining the minimum number of equations required to generate a monomial ideal up to radical (called the arithmetic rank of the ideal) was first studied by P. Schenzel and W. Vogel [11], T. Schmitt and Vogel [12] and G. Lyubeznik [8]. Lyubeznik showed that for a square-free monomial ideal $I$, ara $I \geq \operatorname{pd} R / I$ [8, Proposition 3]. Upper bounds for arithmetic rank have also been considered by M. Barile [1] and [2], building on the work of Schmitt and Vogel mentioned above. In [7], K. Kimura, N. Terai and K.-i. Yoshida raise the question of equality of ara $I$ and $\mathrm{pd} R / I$, and answer it in some cases [7, Theorem 1.1]. It is known, however, due to Z. Yan [17, Example 2] that, in general, $\operatorname{pd} R / I$ and ara $I$ need not be equal.

If $G$ is an unmixed bipartite graph, then we can construct a maximal subgraph $\breve{G}$ which is Cohen-Macaulay; this corresponds to taking a maximal directed acyclic subgraph of $\mathfrak{d}_{G}$. If $G$ is, further, Cohen-Macaulay, then $\breve{G}=G$. Let $\breve{I}$ be the edge ideal of $\breve{G}$. We show that

Theorem 1.3 Let $G$ be an unmixed bipartite graph with edge ideal $I$. Then ara $I \leq$ ara $\breve{I}+\operatorname{pd} R / I-$ ht $I$. If further a maximal acyclic subgraph of $\mathfrak{d}_{G}$ can be embedded in $\mathbb{N}^{2}$, then ara $I=\operatorname{pd} R / I$.

Thus, if $G$ is Cohen-Macaulay and $\mathfrak{d}_{G}$ has an embedding in $\mathbb{N}^{2}$, then $R / I$ is a set-theoretic complete intersection, i.e., it can be defined by ht $I$ equations.

The next section contains definitions, notation and some preliminary observations. Theorems 1.1 and 1.2 are proved in Section 3. A proof of Theorem 1.3 is presented in Section 4.

\section{Edge ideals}

We fix the following notation: $\mathbb{k}$ is a field, $V$ is a finite set of indeterminates over $\mathbb{k}$, $G$ is a simple graph on $V$ without any isolated vertices and $R=\mathbb{k}[V]$ is a polynomial 
ring. We take $I \subseteq R$ to be a square-free monomial ideal; later, we will assume that $I$ is the edge ideal of $G$. Set $c:=$ ht $I$. References for homological aspects of monomial ideals, for graph theory and for results on posets, respectively, are [9, Part I], [16] and [10, Chapter 3]. We will use "multigraded" and "multidegree" to refer to the grading of $R$ by $\mathbb{N}^{|V|}$ and the degrees in this grading.

The multigraded Betti numbers of $R / I$ are $\beta_{l, \sigma}(R / I):=\operatorname{dim}_{\mathbb{k}} \operatorname{Tor}_{l}^{R}(\mathbb{k}, R / I)_{\sigma}$. For $j \in \mathbb{Z}$, the $\left(\mathbb{N}\right.$-graded) Betti numbers are $\beta_{l, j}:=\operatorname{dim}_{\mathbb{k}} \operatorname{Tor}_{l}^{R}(\mathbb{k}, R / I)_{j}$. We note that $\beta_{l, j}(\cdot)=\sum \beta_{l, \sigma}(\cdot)$, where the sum is taken over the set of $\sigma$ with $|\sigma|=j$. (Here |.| denotes the total degree of a multidegree.) To represent a multidegree, we will often use the unique monomial in $R$ of that multidegree; further, if that monomial is square-free, we will use its support, i.e., the set of variables dividing it.

Let $\Delta$ be the Stanley-Reisner complex of $I$. The correspondence between nonfaces of $\Delta$ and monomials in $I$ can also be expressed as follows: for any monomial prime ideal $\mathfrak{p} \in \operatorname{Spec} R, I \subseteq \mathfrak{p}$ if and only if $\mathfrak{p}=(\bar{F}) R$, the ideal generated by $\bar{F}:=V \backslash F$, for some $F \in \Delta[9$, Theorem 1.7]. Thus minimal prime ideals of $R / I$ correspond to complements of maximal faces of $\Delta$. The Alexander dual of $\Delta$, denoted $\Delta^{\star}$, is the simplicial complex $\{\bar{F}: F \notin \Delta\}$. Let $m \in \mathbb{N}$ and $F_{i} \subseteq V, 1 \leq i \leq m$ be such that $\prod_{x \in F_{i}} x, 1 \leq i \leq m$ are the minimal monomial generators of $I$. The Alexander dual of $I$, denoted $I^{\star}$, is the square-free monomial ideal $\bigcap_{i=1}^{m}\left(F_{i}\right)$. If $I$ is the Stanley-Reisner ideal of $\Delta$, then $\bar{F}_{i}, 1 \leq i \leq m$ are precisely the facets of $\Delta^{\star}$. Hence $I^{\star}$ is the Stanley-Reisner ideal of $\Delta^{\star}$. We will need the following theorem of Terai:

Proposition 2.1 (Terai [13]; [9, Theorem 5.59]) For any square-free monomial ideal $J, \operatorname{pd} R / J=\operatorname{reg} J^{\star}$.

The relation between simplicial homology and multigraded Betti numbers is given by Hochster's Formula [9, Corollary 5.12 and Corollary 1.40]. For $\sigma \subseteq V$, we denote by $\left.\Delta\right|_{\sigma}$ the simplicial complex obtained by taking all the faces of $\Delta$ whose vertices belong to $\sigma$. Note that $\left.\Delta\right|_{\sigma}$ is the Stanley-Reisner complex of the ideal $I \cap \mathbb{k}[\sigma]$. Similarly, define the link, $\operatorname{lk}_{\Delta}(\sigma)$, of $\sigma$ in $\Delta$ to be the simplicial complex $\{F \backslash \sigma: F \in$ $\Delta, \sigma \subseteq F\}$. Its Stanley-Reisner ideal in $\mathbb{k}[\bar{\sigma}]$ is $(I: \sigma) \cap \mathbb{k}[\bar{\sigma}]$. First, the multidegrees $\sigma$ with $\beta_{l, \sigma}(R / I) \neq 0$ are square-free. Secondly, for all square-free multidegrees $\sigma$,

$$
\begin{aligned}
\beta_{l, \sigma}(R / I) & =\operatorname{dim}_{\mathbb{k}} \widetilde{H}_{|\sigma|-l-1}\left(\left.\Delta\right|_{\sigma} ; \mathbb{k}\right), \quad \text { and } \\
\beta_{l, \sigma}\left(I^{\star}\right) & =\operatorname{dim}_{\mathbb{k}} \widetilde{H}_{l-1}\left(\operatorname{lk}_{\Delta}(\bar{\sigma}) ; \mathbb{k}\right) .
\end{aligned}
$$

Combining these two formulas we see that

$$
\beta_{l, \sigma}\left(I^{\star}\right)=\beta_{|\sigma|-l, \sigma}\left(\frac{R}{(I: \bar{\sigma})}\right) .
$$

We add, parenthetically, that links of faces in Cohen-Macaulay complexes are themselves Cohen-Macaulay.

We now describe how the graded Betti numbers change under restriction to a subset of the variables and under taking colons. 
Lemma 2.2 Let $I \subseteq R=\mathbb{k}[V]$ be a square-free monomial ideal, $x \in V, l, j \in \mathbb{N}$ and $\sigma \subseteq V$ with $|\sigma|=j$.

(a) Let $W \subseteq V$ and $J=(I \cap \mathbb{k}[W]) R$. Then,

$$
\beta_{l, \sigma}(R / J)= \begin{cases}0, & \sigma \nsubseteq W, \\ \beta_{l, \sigma}(R / I), & \sigma \subseteq W .\end{cases}
$$

In particular, $\beta_{l, j}(R / J) \leq \beta_{l, j}(R / I)$.

(b) If $\beta_{l, \sigma}(R /(I: x)) \neq 0$, then $\beta_{l, \sigma}(R / I) \neq 0$ or $\beta_{l, \sigma \cup\{x\}}(R / I) \neq 0$.

Proof (a): The second assertion follows from the first, which we now prove. Let $\tilde{\Delta}$ be the Stanley-Reisner complex of $J$. Since for all $x \in V \backslash W, x$ does not belong to any minimal prime ideal of $R / J$, we see that every maximal face of $\tilde{\Delta}$ contains $V \backslash W$. Hence if $\sigma \nsubseteq W$, then for all $x \in \sigma \backslash W,\left.\tilde{\Delta}\right|_{\sigma}$ is a cone with vertex $x$, which, being contractible, has zero reduced homology. Applying (1), we see that $\beta_{l, \sigma}(R / J)=0$.

Now let $\sigma \subseteq W$ and $F \subseteq V$. Then $\left.F \in \Delta\right|_{\sigma}$ if and only if $I \subseteq(\bar{F}) R$ and $F \subseteq \sigma$, which holds if and only if $\bar{J} \subseteq(\bar{F}) R$ and $F \subseteq \sigma$, which, in turn, holds if and only if $\left.F \in \tilde{\Delta}\right|_{\sigma}$. Apply (1) again to get

$$
\beta_{l, \sigma}(R / J)=\widetilde{H}_{|\sigma|-l-1}\left(\left.\tilde{\Delta}\right|_{\sigma} ; \mathbb{k}\right)=\widetilde{H}_{|\sigma|-l-1}\left(\left.\Delta\right|_{\sigma} ; \mathbb{k}\right)=\beta_{l, \sigma}(R / I) .
$$

(b): We take the multigraded exact sequence of $R$-modules:

$$
0 \longrightarrow \frac{R}{(I: x)}(-x) \longrightarrow \frac{R}{I} \longrightarrow \frac{R}{(I, x)} \longrightarrow 0
$$

The corresponding multigraded long exact sequence of Tor is

$$
\begin{aligned}
& \cdots \longrightarrow \operatorname{Tor}_{l+1}\left(\mathbb{k}, \frac{R}{(I, x)}\right) \longrightarrow \operatorname{Tor}_{l}\left(\mathbb{k}, \frac{R}{(I: x)}(-x)\right) \\
& \longrightarrow \operatorname{Tor}_{l}\left(\mathbb{k}, \frac{R}{I}\right) \longrightarrow \cdots
\end{aligned}
$$

Let $W=V \backslash\{x\}$ and $J=(I \cap \mathbb{k}[W]) R$. Since $\beta_{l, \sigma}(R /(I: x)) \neq 0$ and $x$ does not divide any monomial minimal generator of $(I: x)$, we have, by the same argument as in (2.2), $\sigma \subseteq W$. Let $\tau=\sigma \cup\{x\}$. First observe that

$$
\operatorname{Tor}_{l}\left(\mathbb{k}, \frac{R}{(I: x)}\right)_{\sigma} \simeq \operatorname{Tor}_{l}\left(\mathbb{k}, \frac{R}{(I: x)}(-x)\right)_{\tau} .
$$

Let us assume that $\beta_{l, \tau}(R / I)=0$, because, if $\beta_{l, \tau}(R / I) \neq 0$, there is nothing to prove. Then, the above long exact sequence of Tor, restricted to the multidegree $\tau$, implies that $\operatorname{Tor}_{l+1}\left(\mathbb{k}, \frac{R}{(I, x)}\right)_{\tau} \neq 0$. Now, since $(I, x)=(J, x)$, we see further $\operatorname{Tor}_{l+1}\left(\mathbb{k}, \frac{R}{(J, x)}\right)_{\tau} \neq 0$.

Since $x$ is a non-zerodivisor on $R / J$, we have a multigraded short exact sequence

$$
0 \longrightarrow \frac{R}{J}(-x) \longrightarrow \frac{R}{J} \longrightarrow \frac{R}{(J, x)} \longrightarrow 0
$$


which gives the following long exact sequence of Tor:

$$
\begin{aligned}
\cdots \longrightarrow \operatorname{Tor}_{l+1}\left(\mathbb{k}, \frac{R}{J}\right) \longrightarrow & \operatorname{Tor}_{l+1}\left(\mathbb{k}, \frac{R}{(J, x)}\right) \\
\longrightarrow & \operatorname{Tor}_{l}\left(\mathbb{k}, \frac{R}{J}(-x)\right) \longrightarrow \cdots
\end{aligned}
$$

Since $x$ does not divide any minimal monomial generator of $J, \beta_{l+1, \tau}(R / J)=0$. Therefore $\operatorname{Tor}_{l}\left(\mathbb{k}, \frac{R}{J}(-x)\right)_{\tau} \neq 0$, or, equivalently, $\operatorname{Tor}_{l}\left(\mathbb{k}, \frac{R}{J}\right)_{\sigma} \neq 0$. By (2.2) above, $\beta_{l, \sigma}(R / I) \neq 0$.

If $\mathfrak{p} \subseteq R$ is a prime ideal such that ht $\mathfrak{p}=c=$ ht $I$ and $I \subseteq \mathfrak{p}$, then we say that $\mathfrak{p}$ is an unmixed associated prime ideal of $R / I$. Denote the set of unmixed associated prime ideals of $R / I$ by Unm $R / I$. Unmixed prime ideals are necessarily minimal over $I$, so Unm $R / I \subseteq$ Ass $R / I$; we say that $I$ or $R / I$ is unmixed if $\operatorname{Unm} R / I=$ Ass $R / I$.

We now restrict our attention to edge ideals of graphs. Every square-free quadratic monomial ideal can be considered as the edge ideal of some simple graph. The theory of edge ideals is systematically developed in [14, Chapter 6]. Hereafter $I$ is the edge ideal of $G$, which we have set to be a simple graph on $V$. A vertex cover of $G$ is a set $A \subseteq V$ such that whenever $x y$ is an edge of $G, x \in A$ or $y \in A$. It is easy to see that for all $A \subseteq V, A$ is a vertex cover of $G$ if and only if the prime ideal $(x: x \in A)$ contains $I$. Since $I$ is square-free, $R / I$ is reduced; therefore, Ass $R / I$ is the set of minimal prime ideals containing $I$. These are monomial ideals, and, hence, are in bijective correspondence with the set of minimal vertex covers of $G$. We will say that $G$ is unmixed (respectively, Cohen-Macaulay) if $R / I$ is unmixed (respectively, Cohen-Macaulay). Observe that if $G$ is unmixed, then all its minimal vertex covers have the same size.

If $x y$ is an edge of $G$, then we say that $x$ and $y$ are neighbours of each other. An edge is incident on its vertices. We say that an edge $x y$ is isolated if there are no other edges incident on $x$ or on $y$. Let $G$ be a graph. A matching in $G$ is a maximal (under inclusion) set $\mathrm{m}$ of edges such that for all $x \in V$, at most one edge in $\mathrm{m}$ is incident on $x$. Edges in a matching form a regular sequence on $R$. We say that $G$ has perfect matching, or, is perfectly matched, if there is a matching $\mathrm{m}$ such that for all $x \in V$, exactly one edge in $\mathrm{m}$ is incident on $x$.

Lemma 2.3 Let $G$ be a bipartite graph on the vertex set $V=V_{1} \bigsqcup V_{2}$, with edge ideal I. Then $G$ has a perfect matching if and only if $\left|V_{1}\right|=\left|V_{2}\right|=\mathrm{ht} I$. In particular, unmixed bipartite graphs have perfect matching.

Proof If $G$ has a perfect matching, then $\left|V_{1}\right|=\left|V_{2}\right|$. Moreover, by König's theorem [16, Theorem 3.1.16], the maximum size of any matching equals the minimum size of any vertex cover; hence $\left|V_{1}\right|=\left|V_{2}\right|=$ ht $I$. Conversely, if $\left|V_{1}\right|=\left|V_{2}\right|=$ ht $I$, then, again by König's theorem, $G$ has a matching of $\left|V_{1}\right|=\left|V_{2}\right|$ edges, i.e., it has a perfect matching.

If $G$ is unmixed, then every minimal vertex cover of $G$ has the same size. Observe that both $V_{1}$ and $V_{2}$ are minimal vertex covers of $G$. 
Discussion 2.4 Let $\mathfrak{d}$ be any directed graph on $[c]$, and denote the underlying undirected graph of $\mathfrak{d}$ by $|\mathfrak{d}|$. We will write $j \succ i$ if there is a directed path from $i$ to $j$ in $\mathfrak{d}$. By $j \succcurlyeq i$ (and, equivalently, $i \preccurlyeq j$ ) we mean that $j \succ i$ or $j=i$. For $A \subseteq[c]$, we say that $j \succcurlyeq A$ if there exists $i \in A$ such that $j \succcurlyeq i$. We say that a set $A \subseteq[c]$ is an antichain if for all $i, j \in A$, there is no directed path from $i$ to $j$ in $\mathfrak{d}$, and, by $\mathcal{A}_{\mathfrak{d}}$, denote the set of antichains in $\mathfrak{d}$. We consider $\varnothing$ as an antichain. A coclique of $|\mathfrak{d}|$ is a set $A \subseteq[c]$ such that for all $i \neq j \in A, i$ and $j$ are not neighbours in $|\mathfrak{d}|$. Antichains in $\mathfrak{d}$ are cocliques in $|\mathfrak{d}|$, but the converse is not, in general, true. We say that $\mathfrak{d}$ is acyclic if there are no directed cycles, and transitively closed if, for all $i, j, k \in[c]$, whenever $i j$ and $j k$ are (directed) edges in $\mathfrak{d}, i k$ is an edge. Observe that $\mathfrak{d}$ is a poset under the order $\succcurlyeq$ if and only if it is acyclic and transitively closed. If $\mathfrak{d}$ is a poset, we say that, for $i, j \in[c], j$ covers $i$ if $j \succ i$ and there does not exist $j^{\prime}$ such that $j \supsetneqq j^{\prime} \varsubsetneqq i$. Let $G$ be a bipartite graph on $V=V_{1} \bigsqcup V_{2}$ with perfect matching. Let $V_{1}=\left\{x_{1}, \cdots, x_{c}\right\}$ and $V_{2}=\left\{y_{1}, \cdots, y_{c}\right\}$. After relabelling the vertices, we will assume that $x_{i} y_{i}$ is an edge for all $i \in[c]$. We associate $G$ with a directed graph $\mathfrak{d}_{G}$ on $[c]$ defined as follows: for $i \neq j \in[c], i j$ is an edge of $\mathfrak{d}_{G}$ if and only if $x_{i} y_{j}$ is an edge of $G$. (Here, by $i j$, we mean the directed edge from $i$ to $j$.) Observe that $\mathfrak{d}_{G}$ is simple, i.e., without loops and multiple edges. Let $\kappa(G)$ denote the largest size of any coclique in $\left|\mathfrak{d}_{G}\right|$.

The significance of $\kappa(G)$ is that it gives a lower bound for reg $R / I$. Following Zheng [19], we say that two edges $v w$ and $v^{\prime} w^{\prime}$ of a graph $G$ are disconnected if they are no more edges between the four vertices $v, v^{\prime}, w, w^{\prime}$. A set $\mathbf{a}$ of edges is pairwise disconnected if and only if $\left(I \cap \mathbb{k}\left[V_{\mathbf{a}}\right]\right) R$ is generated by the regular sequence of edges in $\mathbf{a}$, where by $V_{\mathbf{a}}$, we mean the set of vertices on which the edges in $\mathbf{a}$ are incident. The latter condition holds if and only if the subgraph of $G$ induced on $V_{\mathbf{a}}$, denoted as $\left.G\right|_{V_{\mathbf{a}}}$, is a collection of $|\mathbf{a}|$ isolated edges. In particular, the edges in any pairwise disconnected set form a regular sequence in $R$. Set $r(I):=\max \{|\mathbf{a}|$ : $\mathbf{a}$ is a set of pairwise disconnected edges in $G$ \}.

Lemma 2.5 Let $G$ be bipartite graph with perfect matching. Then, with notation as in Discussion 2.4, $r(I) \geq \kappa(G) \geq \max \left\{|A|: A \in \mathcal{A}_{\mathfrak{d}_{G}}\right\}$.

Proof If $A \subseteq[c]$ is a coclique of $\left|\mathfrak{d}_{G}\right|$, we easily see that the edges $\left\{x_{i} y_{i}: i \in A\right\}$ are pairwise disconnected in $G$. The assertion now follows from the observation, which we made in Discussion 2.4, that any antichain in $\mathfrak{d}_{G}$ is a coclique of $\left|\mathfrak{d}_{G}\right|$.

The assertion of Theorem 1.1 is that when $G$ is an unmixed bipartite graph, equality holds in the above lemma and that this quantity equals reg $R / I$. We will prove Theorem 1.1 in the next section; now, we relate some properties of bipartite graphs with their associated directed graphs.

Lemma 2.6 Let $G$ be bipartite graph with perfect matching, and adopt the notation of Discussion 2.4. Let $j \succcurlyeq i$. Then for all $\mathfrak{p} \in \operatorname{Unm} R / I$, if $y_{i} \in \mathfrak{p}$, then $y_{j} \in \mathfrak{p}$.

Proof Applying induction on the length of a directed path from $i$ to $j$, we may assume, without loss of generality, that $i j$ is a directed edge of $\mathfrak{d}_{G}$. Let $\mathfrak{p} \in \operatorname{Unm} R / I$ 
and $k \in[c]$. Since $x_{k} y_{k} \in I, x_{k} \in \mathfrak{p}$ or $y_{k} \in \mathfrak{p}$. Since ht $\mathfrak{p}=c$, in fact, $x_{k} \in \mathfrak{p}$ if and only if $y_{k} \notin \mathfrak{p}$. Now since $y_{i} \in \mathfrak{p}, x_{i} \notin \mathfrak{p}$, so $\left(I: x_{i}\right) \subseteq \mathfrak{p}$. Note that since $x_{i} y_{j}$ is an edge of $G, y_{j} \in\left(I: x_{i}\right)$.

Theorem 2.7 Let $G$ be a bipartite graph on the vertex set $V=V_{1} \bigsqcup V_{2}$.

(a) $\left[15\right.$, Theorem 1.1] $G$ is unmixed if and only if $G$ has a perfect matching and $\mathfrak{d}_{G}$ is transitively closed.

(b) [4, Lemma 3.3 and Theorem 3.4] $G$ is Cohen-Macaulay if and only if $G$ is perfectly matched and the associated directed graph $\mathfrak{d}_{G}$ is acyclic and transitively closed, i.e., it is a poset.

Discussion 2.8 Let $\mathfrak{d}$ be a directed graph. We say that a pair $i, j$ of vertices $\mathfrak{d}$ are strongly connected if there are directed paths from $i$ to $j$ and from $j$ to $i$; see [16, Definition 1.4.12]. A strong component of $\mathfrak{d}$ is an induced subgraph maximal under the property that every pair of vertices in it is strongly connected. Strong components of $\mathfrak{d}$ form a partition of its vertex set. Now let $G$ be a bipartite graph with perfect matching. Let $\mathcal{Z}_{1}, \ldots, \mathcal{Z}_{t}$ be the vertex sets of the strong components of $\mathfrak{d}_{G}$. Define a directed graph $\widehat{\mathfrak{d}}$ on $[t]$ by setting, for $a \neq b \in[t], a b$ to be a directed edge (from $a$ to $b$ ) if there exists a directed path in $\mathfrak{d}_{G}$ from any (equivalently, all, since $\mathfrak{d}_{G} \mid \mathcal{Z}_{a}$ is strongly connected) of the vertices in $\mathcal{Z}_{a}$ to any (equivalently, all, since $\left.\mathfrak{d}_{G}\right|_{\mathcal{Z}_{b}}$ is transitively closed) of the vertices in $\mathcal{Z}_{b}$. We observe that $\widehat{\mathfrak{d}}$ has no directed cycles. Now assume further that $G$ is unmixed. Then, since $\mathfrak{d}_{G}$ is transitively closed, $\widehat{\mathfrak{d}}$ is transitively closed, i.e., it is a poset under the order induced from $\mathfrak{d}_{G}$. We will use the same notation for the induced order, i.e., say that $b \succ a$ if there is a directed edge from $a$ to $b$. Define the acyclic reduction of $G$ to be the bipartite graph $\widehat{G}$ on new vertices $\left\{u_{1}, \ldots, u_{t}\right\} \bigsqcup\left\{v_{1}, \ldots, v_{t}\right\}$, with edges $u_{a} v_{a}$, for all $1 \leq a \leq t$ and $u_{a} v_{b}$, for all directed edges $a b$ of $\widehat{\mathfrak{d}}$. Let $S=\mathbb{k}\left[u_{1}, \ldots, u_{t}, v_{1}, \ldots, v_{t}\right]$, with standard grading. Let $\widehat{I} \subseteq S$ be the edge ideal of $\widehat{G}$. Let $\zeta_{i}=\left|\mathcal{Z}_{i}\right|, 1 \leq i \leq t$. For a multidegree $\tau=\prod_{i} u_{i}^{s_{i}} \prod v_{i}^{t_{i}}$, set $\tau^{\zeta}=\prod_{i} u_{i}^{s_{i} \zeta_{i}} \prod v_{i}^{t_{i} \zeta_{i}}$.

Lemma 2.9 Let $G$ be an unmixed bipartite graph with edge ideal I. For an antichain $A \neq \varnothing$ of $\widehat{\mathfrak{d}}$, let $\Omega_{A}=\left\{j \in \mathcal{Z}_{b}: b \succcurlyeq A\right\}$. Let $\Omega_{\varnothing}=\varnothing$. Then Ass $R / I=\left\{\left(x_{i}: i \notin\right.\right.$ $\left.\left.\Omega_{A}\right)+\left(y_{i}: i \in \Omega_{A}\right): A \in \mathcal{A}_{\widehat{d}}\right\}$.

Proof Let $\mathfrak{p} \in$ Ass $R / I$. Let $\mathrm{U}:=\left\{b: y_{j} \in \mathfrak{p}\right.$ for some $\left.j \in \mathcal{Z}_{b}\right\}$. It follows from Lemma 2.6 that $y_{j} \in \mathfrak{p}$ for all $j \in \bigcup_{b \in \mathrm{U}} \mathcal{Z}_{b}$ and that if $b^{\prime} \succ b$ for some $b \in \mathrm{U}$, then $b^{\prime} \in \mathrm{U}$. Now, the minimal elements of $U$ form an antichain $A$ under $\succ$. Hence $\left\{j: y_{j} \in \mathfrak{p}\right\}=\Omega_{A}$, showing Ass $R / I \subseteq\left\{\left(x_{i}: i \notin \Omega_{A}\right)+\left(y_{i}: i \in \Omega_{A}\right): A \in \mathcal{A}_{\widehat{d}}\right\}$.

Conversely, let $A \in \mathcal{A}_{\widehat{\mathfrak{d}}}$ and $\mathfrak{p}:=\left(x_{i}: i \notin \Omega_{A}\right)+\left(y_{i}: i \in \Omega_{A}\right)$. Since ht $\mathfrak{p}=c=$ ht $I$, it suffices to show that $I \subseteq \mathfrak{p}$ in order to show that $\mathfrak{p} \in$ Ass $R / I$. Clearly, for all $1 \leq i \leq c, x_{i} y_{i} \in \mathfrak{p}$. Take $i \neq j$ such that $x_{i} y_{j} \in I$. If $i \notin \Omega_{A}$, then there is nothing to be shown. If $i \in \Omega_{A}$, then there exist $a, b, b^{\prime}$ such that $a \in A, b \succ a, i \in \mathcal{Z}_{b}$ and $j \in \mathcal{Z}_{b^{\prime}}$. Since $i j$ is a directed edge of $\mathfrak{d}_{G}, b^{\prime} \succ b$ in $\widehat{\mathfrak{d}}$. Hence $b^{\prime} \succ a$, and $j \in \Omega_{A}$, giving $y_{j} \in \mathfrak{p}$. This shows that $I \subseteq \mathfrak{p}$. 


\section{Regularity and depth}

The content of Lemma 2.9 is that there are subsets $W \subseteq V$ such that for all $\mathfrak{p} \in$ Ass $R / I$, if $\mathfrak{p} \cap W \neq \varnothing$ then $W \subseteq \mathfrak{p}$. Looking at $I^{\star}$, we see that for all minimal generators $g$ of $I^{\star}$, if any element of $W$ divides $g$, then all elements of $W$ divide $g$. Label the minimal monomial generators of $I^{\star}$ as $g_{1}, \ldots, g_{s}, g_{s+1}, \ldots, g_{m}$ so that every element of $W$ divides $g_{1}, \ldots, g_{s}$ and no element of $W$ divides $g_{s+1}, \ldots, g_{m}$. Fix $x \in W$. For $i=1, \ldots, s$, set $h_{i}:=\frac{x^{|W|}}{\prod_{y \in W} y} g_{i}$ and $\bar{h}_{i}:=\frac{x}{\prod_{y \in W} y} g_{i}$. Let $J=$ $\left(h_{1}, \ldots, h_{s}, g_{s+1}, \ldots, g_{m}\right)$ and $J^{\prime}=\left(\bar{h}_{1}, \ldots, \bar{h}_{s}, g_{s+1}, \ldots, g_{m}\right)$. Let $\phi: R \rightarrow R$ be the ring homomorphism that sends $x \mapsto x^{|W|}$ and $y \mapsto y$, for all $y \neq x \in V$. We make two observations: first, that $I^{\star}$ is a polarization of $J$, and, secondly, that $J=\phi\left(J^{\prime}\right)$. Hence the $\mathbb{N}$-graded Betti numbers of $I^{\star}$ and $J$ are identical [9, Exercise 3.15]. Further, the following lemma shows that $\beta_{l, \sigma}(R / J) \neq 0$ if and only if $x^{|W|}$ divides $\sigma$ and $\beta_{l, \frac{\sigma}{x^{|W|-1}}}(R / J) \neq 0$.

Lemma 3.1 Let $B_{1}=\mathbb{k}\left[x_{1}, \ldots, x_{n}\right]$ and $B_{2}=\mathbb{k}\left[y_{1}, \ldots, y_{n}\right]$. Let $\xi_{1}, \ldots, \xi_{n}$ be positive integers. Set $\operatorname{deg} x_{i}=1$ and $\operatorname{deg} y_{i}=\xi_{n}$ for all $1 \leq i \leq n$. Define a ring homomorphism $\phi: B_{2} \rightarrow B_{1}$ by sending $y_{i} \mapsto x_{i}^{\xi_{i}}$. Then for any acyclic complex $\mathbb{G}_{\bullet}$ of finitely generated graded $B_{2}$-modules (with degree-preserving maps), $\mathbb{G}_{\bullet} \otimes_{B_{2}} B_{1}$ is an acyclic complex of finitely generated graded $B_{1}$-modules (with degree-preserving maps).

Proof Acyclicity of $\mathbb{G}_{\bullet} \otimes_{B_{2}} B_{1}$ follows from the fact that $B_{1}$ is a free and hence flat $B_{2}$-algebra. The maps in $\mathbb{G}_{\bullet} \otimes_{B_{2}} B_{1}$ are degree-preserving since $\phi$ preserves degrees.

Proposition 3.2 Let $G$ be an unmixed bipartite graph, with edge ideal I and acyclic reduction $\widehat{G}$. Let $\widehat{I} \subseteq S$ be the edge ideal of $\widehat{G}$. Then $\operatorname{reg} R / I=\operatorname{pd}(\widehat{I})^{\star}$ and $\operatorname{pd} R / I=$ $\max \left\{\left|\tau^{\zeta}\right|-l: \beta_{l, \tau}\left((\widehat{I})^{\star}\right) \neq 0\right\}$.

Proof By Proposition 2.1, $\operatorname{reg} R / I=\operatorname{pd} I^{\star}$ and $\operatorname{pd} R / I=\operatorname{reg} I^{\star}$. Hence it suffices to show that $\operatorname{pd} I^{\star}=\operatorname{pd}(\widehat{I})^{\star}$ and $\operatorname{reg} I^{\star}=\max \left\{\left|\sigma^{\zeta}\right|-l: \beta_{l, \sigma}\left((\widehat{I})^{\star}\right) \neq 0\right\}$. From Lemma 2.9, with the notation used there, it follows that

$$
I^{\star}=\left(\prod_{i \notin \Omega_{A}} x_{i} \cdot \prod_{i \in \Omega_{A}} y_{i}: A \in \mathcal{A}_{\widehat{\mathfrak{d}}}\right)=\left(\prod_{\substack{b \neq A \\ i \in \mathcal{Z}_{b}}} x_{i} \cdot \prod_{\substack{b \succcurlyeq A \\ i \in \mathcal{Z}_{b}}} y_{i}: \varnothing \neq A \in \mathcal{A}_{\widehat{\mathfrak{d}}}\right)+\left(\prod_{i=1}^{c} x_{i}\right) .
$$

For each $a \in[t]$, fix $i_{a} \in \mathcal{Z}_{a}$. Now, as the $\mathcal{Z}_{a}$ form a partition of $[c]$, we see that $I^{\star}$ is a polarization of the ideal

$$
J=\left(\prod_{b \nsucceq A} x_{i_{b}}^{\zeta_{b}} \cdot \prod_{b \succcurlyeq A} y_{i_{b}}^{\zeta_{b}}: \varnothing \neq A \in \mathcal{A}_{\widehat{\mathfrak{d}}}\right)+\left(\prod_{b=1}^{t} x_{i_{b}}^{\zeta_{b}}\right)
$$




$$
\subseteq S:=\mathbb{k}\left[x_{i_{1}}, \ldots, x_{i_{t}}, y_{i_{1}}, \ldots, y_{i_{t}}\right] .
$$

Notice that $S^{\prime} \simeq S$ (which, we recall, is the polynomial ring on the vertex set of the acyclic reduction $\widehat{G}$ ) under the map $\phi: x_{i_{a}} \mapsto u_{a}$ and $\phi: y_{i_{a}} \mapsto v_{a}$, and that $\phi(\sqrt{J})=(\widehat{I})^{\star}$. Therefore $\beta_{l, \sigma}(\sqrt{J})=\beta_{l, \phi(\sigma)}\left((\widehat{I})^{\star}\right)$. It now suffices to show that $\operatorname{pd} J=\operatorname{pd} \sqrt{J}$ and that $\operatorname{reg} J=\max \left\{\left|\tau^{\zeta}\right|-l: \beta_{l, \tau}(\sqrt{J}) \neq 0\right\}$. This, being the same argument as in the opening paragraph of this section, follows from the preceding lemma.

Remark 3.3 Let $G$ be an unmixed graph with acyclic reduction $\widehat{G}$. If $I \subseteq R$ and $\widehat{I} \subseteq S$ are the respective edge ideals, then it follows from Proposition 3.2 that $\operatorname{reg} R / I=$ $\operatorname{pd}(\widehat{I})^{\star}=\operatorname{reg} S / \widehat{I}$.

Lemma 3.4 Let $G$ be an unmixed bipartite graph with acyclic reduction $\widehat{G}$. Then $\max \left\{|A|: A \in \mathcal{A}_{\mathfrak{d}_{G}}\right\}=\max \left\{|A|: A \in \mathcal{A}_{\mathfrak{d}_{\widehat{G}}}\right\}$.

Proof Let $A=\left\{i_{1}, \ldots, i_{r}\right\} \subseteq[c]$ be an antichain in $\mathfrak{d}_{G}$. Choose $a_{1}, \ldots, a_{r} \in[t]$ such that $i_{j} \in \mathcal{Z}_{a_{j}}$. Since $\mathfrak{d}_{G}$ is transitively closed, it follows that $\left\{a_{1}, \ldots, a_{r}\right\}$ is an antichain in $\mathfrak{d}_{\widehat{G}}$. Conversely, if $\left\{a_{1}, \ldots, a_{r}\right\}$ is an antichain in $\mathfrak{d}_{\widehat{G}}$, then for any choice of $i_{j} \in \mathcal{Z}_{a_{j}},\left\{i_{1}, \ldots, i_{r}\right\}$ is an antichain in $\mathfrak{d}_{G}$.

We now prove Theorem 1.1. If $G$ is a tree — trees are bipartite - then reg $R / I$ is the maximum size of a pairwise disconnected set of edges in $G$, without the assumption that $G$ is unmixed [19, Theorem 2.18]. However, for bipartite graphs $G$ that are not trees, we need to assume that $G$ is unmixed. For example, if $G$ is the cycle on eight vertices, we can choose at most two edges that are pairwise disconnected, while $\operatorname{reg} R / I=3$.

Theorem 1.1 Let $G$ be an unmixed bipartite graph with edge ideal I. Then $\operatorname{reg} R / I=\max \left\{|A|: A\right.$ is an antichain in $\left.\mathfrak{d}_{G}\right\}$. In particular, $\operatorname{reg} R / I$ is the maximum size of a pairwise disconnected set of edges in $G$.

Proof Since reg $R / I \geq r(I)$ (see the paragraph on page 429 following the statement of Theorem 1.1), the latter statement follows from the first statement along with Lemma 2.5 . In order to prove the first statement, let $\widehat{G}$ be the acyclic reduction of $G$ on the vertex set $\left\{u_{1}, \ldots, u_{t}\right\} \bigsqcup\left\{v_{1}, \ldots, v_{t}\right\}$. Recall that $\widehat{G}$ is a Cohen-Macaulay bipartite graph. As in Discussion 2.8 , let $S=\mathbb{k}\left[u_{1}, \ldots, u_{t}, v_{1}, \ldots, v_{t}\right]$. Let $\widehat{I} \subseteq S$ be the edge ideal of $\widehat{G}$. Remark 3.3 and Lemma 3.4 give that it suffices to prove the theorem for Cohen-Macaulay bipartite graphs. If $G$ is Cohen-Macaulay, then $\mathfrak{d}_{G}$ is a poset. From [4, Corollary 2.2], taken along with Proposition 2.1, we see that $\operatorname{pd} R / I=\max \left\{|A|: A \in \mathcal{A}_{\mathfrak{d}_{G}}\right\}$. (Note that $I^{\star}$ is the ideal $H_{\mathfrak{d}_{G}}$, in the notation of [4], with the $x_{i}$ and the $y_{j}$ interchanged.)

Remark 3.5 Let $G$ be a Cohen-Macaulay bipartite graph with edge ideal $I$, with ht $I=c$. Then $\operatorname{reg} R / I \leq c$. If $\operatorname{reg} R / I=c$, then $R / I$ is a complete intersection, or, equivalently, $G$ consists of $c$ isolated edges. We see this as below: Let $\mathfrak{d}_{G}$ be the 
associated directed graph on $[c]$. Since reg $R / I$ is the maximum size of an antichain in $\mathfrak{d}_{G}, \operatorname{reg} R / I \leq c$. If $\operatorname{reg} R / I=c$, we see that $\mathfrak{d}_{G}$ has an antichain of $c$ elements, which implies that for all $i \neq j \in[c], i \neq j$ or $j \neq i$, i.e., $x_{i} y_{j}$ is not an edge of $G$.

We would now like to give a description of depth $R / I$ for an unmixed bipartite edge ideal $I$ in terms of the associated directed graph. First, we determine the multidegrees with non-zero Betti numbers for its Alexander dual. Let $G$ be a Cohen-Macaulay bipartite graph. For antichains $B \subseteq A$ of $\mathfrak{d}_{G}, A \neq \varnothing$, set $\sigma_{A, B}:=$ $\prod_{i \nsucc A} x_{i} \prod_{i \succcurlyeq A} y_{i} \prod_{i \in B} x_{i}$. Set $\sigma_{\varnothing, \varnothing}=\prod_{i=1}^{c} x_{i}$. With this notation, we restate [4, Theorem 2.1] as follows:

Theorem 3.6 Let $G$ be a Cohen-Macaulay bipartite graph with edge ideal I. For all $l \geq 0$, and multidegrees $\sigma$, if $\beta_{l, \sigma}\left(I^{\star}\right) \neq 0$, then $\beta_{l, \sigma}\left(I^{\star}\right)=1$ and $\sigma=\sigma_{A, B}$ for some antichains $B \subseteq A$ of $\mathfrak{d}_{G}$ with $|B|=l$.

(Although the multidegrees in which the Betti numbers are non-zero are not explicitly given in the statement of [4, Theorem 2.1], we can determine them easily from the description of the differentials given there, prior to stating the theorem. Note, again, that the roles of the $x_{i}$ and the $y_{j}$ are the opposite of what we follow.)

Corollary 3.7 Let $G$ be an unmixed bipartite graph with edge ideal I. Let $c=$ ht $I$. Let $t, \zeta_{1}, \ldots, \zeta_{t}, \widehat{\mathfrak{d}}$ be as in Discussion 2.8. Then

$$
\operatorname{depth} R / I=c-\max \left\{\sum_{i \in B} \zeta_{i}-|B|: B \text { is an antichain of } \widehat{\mathfrak{d}}\right\} \text {. }
$$

Proof Let $\widehat{G}, S, \widehat{I}$ be as in Discussion 2.8. From Theorem 3.6, we know that if $\beta_{l, \sigma}\left((\widehat{I})^{\star}\right) \neq 0$ for some multidegree $\sigma \subseteq\left\{u_{1}, \ldots, u_{t}, v_{1}, \ldots, v_{t}\right\}$, then $\sigma=\sigma_{A, B}$ for some antichains $B \subseteq A$ of $\widehat{\mathfrak{d}}$, with $|\bar{B}|=l$. Now, in $S, \operatorname{deg} \sigma_{A, B}=\sum_{i \succcurlyeq A} \zeta_{i}+$ $\sum_{i \nsucc A} \zeta_{i}+\sum_{i \in B} \zeta_{i}=c+\sum_{i \in B} \zeta_{i}$. Hence

$$
\operatorname{reg}(\widehat{I})^{\star}=c+\max \left\{\sum_{i \in B} \zeta_{i}-|B|: B \text { is an antichain of } \widehat{\mathfrak{d}}\right\} .
$$

Note that $\operatorname{depth} R=\operatorname{dim} R=2 c$. Now apply Proposition 3.2, followed by the Auslander-Buchsbaum formula, to obtain the conclusion.

The above proof also shows that if $G$ is a bipartite graph such that $R / I$ satisfies Serre's condition $\left(S_{2}\right)$ (defined, e.g., in [3, Section 2.1]) then $G$ is Cohen-Macaulay. For, if $R / I$ satisfies $\left(S_{2}\right)$, then it is unmixed and $I^{\star}$ is linearly presented, i.e., the nonzero entries in any matrix giving a presentation of $I^{\star}$ are linear. This is a special case of [18, Corollary 3.7]. It follows, with the notation of the proof, that for all antichains $A \neq \varnothing$ of $\widehat{\mathfrak{d}}$, and for all $a \in A, \operatorname{deg} \sigma_{A,\{a\}}=c+\zeta_{a}=c+1$, giving that every strong component of $\mathfrak{d}_{G}$ has exactly one element. In other words, $G$ is Cohen-Macaulay. We can now prove Theorem 1.2. 
Theorem 1.2 Let $G$ be an unmixed bipartite graph, with edge ideal I and associated directed graph $\mathfrak{d}_{G}$. If $\mathfrak{d}_{G}$ has $t$ strong components, then depth $R / I \geq t$.

Proof To show that depth $R / I \geq t$, it suffices to show that, for all antichains $B$ of $\widehat{\mathfrak{d}}$, $t+\sum_{i \in B} \zeta_{i}-|B| \leq c$. Since $c=\sum_{i=1}^{t} \zeta_{i}$, it suffices to show that $t-|B| \leq \sum_{i \notin B} \zeta_{i}$, which is true since $\zeta_{i} \geq 1$ for all $i$.

Remark 3.8 The above bound is sharp. Given positive integers $t \leq c$, and a poset $\widehat{d}$ on $t$ vertices, we can find an unmixed bipartite graph $G$ on the vertex set $V=V_{1} \bigsqcup V_{2}$ with edge ideal $I$ such that $\left|V_{1}\right|=\left|V_{2}\right|=c$ and depth $\mathbb{k}[V] / I=t$. Choose any antichain $B$ in $\widehat{\mathfrak{d}}$ and set $\zeta_{i}=1$ for all $i \notin B$. Choose $\zeta_{i} \geq 1, i \in B$ such that $\sum_{i \in B} \zeta_{i}=$ $c-t+|B|$. Now construct a directed graph $\mathfrak{d}$ on $c$ vertices by replacing the vertex $i$ of $\widehat{\mathfrak{d}}$ by directed cycle of $\zeta_{i}$ vertices and then taking its transitive closure. Label the vertices of $\mathfrak{d}$ with $[c]$. Let $G$ be a bipartite graph on $V=\left\{x_{1}, \ldots, x_{c}\right\} \bigsqcup\left\{y_{1}, \ldots, y_{c}\right\}$ such that $x_{i} y_{i}$ is an edge for all $i \in[c]$ and $x_{i} y_{j}$ is an edge whenever $i j$ is a directed edge of $\mathfrak{d}$. Then $G$ is an unmixed graph. We know from the corollary that $t \leq \operatorname{depth} R / I \leq c-\sum_{i \in B} \zeta_{i}-|B|=t$.

\section{Arithmetic rank}

The two statements of Theorem 1.3 will be proved separately in Proposition 4.2 and in Proposition 4.11.

Discussion 4.1 Let $G$ be an unmixed bipartite graph on $\left\{x_{1}, \ldots, x_{c}\right\} \bigsqcup\left\{y_{1}, \ldots, y_{c}\right\}$. Adopt the notation of Discussion 2.8. Choose an acyclic transitively closed subgraph of $\mathfrak{d}_{G}$ which is maximal under inclusion of edge sets; call it $\breve{d}$. It is a poset, with the order induced from $\mathfrak{d}_{G}$. We will denote this order by $\triangleright$ to avoid confusion with $\succ$. (Recall that $\succ$ does not define a partial order if $G$ is not Cohen-Macaulay.) Let $\breve{G}$ be the Cohen-Macaulay bipartite graph on $\left\{x_{1}, \ldots, x_{c}\right\} \bigsqcup\left\{y_{1}, \ldots, y_{c}\right\}$ corresponding to $\breve{\mathfrak{d}}$; denote its edge ideal by $\breve{I}$.

Proposition 4.2 With notation as above, ara $I \leq \operatorname{ara} \breve{I}+\operatorname{pd} R / I-\operatorname{ht} I$.

Proof On the set $\left\{x_{j} y_{i}: j \triangleright i, j \neq i\right.$ and $x_{j} y_{i}$ is an edge of $\left.G\right\}$, define a partial order: $x_{j} y_{i}>x_{j^{\prime}} y_{i^{\prime}}$ whenever $j \triangleright j^{\prime}, j \neq j^{\prime}, i \triangleright i^{\prime}, i \neq i^{\prime}$. Call this poset $P$. (These are the edges of $G$ that do not belong to $\breve{G}$. If $x_{j} y_{i}$ is such an edge, then $i$ and $j$ belong to the same strong component of $\mathfrak{d}_{G}$.) We now claim that every antichain in $P$ has at most $\max \left\{\sum_{a \in B} \zeta_{a}-|B|: B\right.$ is an antichain of $\left.\widehat{\mathfrak{d}}\right\}$ elements; this quantity, as we note from Corollary 3.7, equals $\xi:=\operatorname{pd} R / I-$ ht $I$. Let $\left\{x_{j_{k}} y_{i_{k}}: 1 \leq k \leq l\right\}$ with $j_{k} \triangleright i_{k}, 1 \leq k \leq l$ be an antichain in $P$. First, there exist $a_{1}, \ldots, a_{l}$ such that $i_{k}, j_{k} \in$ $\mathcal{Z}_{a_{k}}$; this arises from the fact that $j_{k} \triangleright i_{k}$. If $a_{k_{2}} \nsucceq a_{k_{1}}$, then for, $i, j \in \mathcal{Z}_{a_{k_{1}}}$ and $i^{\prime}, j^{\prime} \in \mathcal{Z}_{a_{k_{2}}}, x_{j^{\prime}} y_{i^{\prime}}>x_{j} y_{i}$, so if $a_{k_{2}} \neq a_{k_{1}}$, then they are incomparable. Therefore, to prove the claim, it suffices to show that if $a_{1}=\ldots=a_{l}=a$, say, then $l \leq \zeta_{a}-1$. This follows easily, for, in this case, any antichain in $P$ can contain at most one edge for each value of $j-i$, and $1 \leq j-i \leq \zeta_{a}-1$. Moreover, let $B$ be an antichain of $\widehat{\mathfrak{d}}$ 
for which the maximum is attained. For all $a \in B$, set $j_{a}$ to be the maximal element of $\mathcal{Z}_{a}$ under $\triangleright$. Then $\left\{x_{j_{a}} y_{i}: i \in \mathcal{Z}_{a}, a \in B\right\}$ is an antichain of $P$ with $\xi$ elements. Using Dilworth's theorem [16, p. 413], we cover $P$ with $\xi$ chains, $\mathcal{C}_{1}, \ldots, \mathcal{C}_{\xi}$. For $1 \leq k \leq \xi$, set $h_{k}:=\sum_{x_{j} y_{i} \in \mathcal{C}_{k}} x_{j} y_{i}$.

Our final claim is that $\sqrt{\breve{I}+\left(h_{1}, \ldots, h_{\xi}\right)}=I$. The $h_{l}$ belong to $I$ and $\breve{I} \subseteq I$, so it suffices to show that $I \subseteq \mathfrak{p}$ for every $\mathfrak{p} \in \operatorname{Spec} R$ such that $\breve{I}+\left(h_{1}, \ldots, h_{\xi}\right) \subseteq \mathfrak{p}$. Let $\mathfrak{p}$ be such, and, by way of contradiction, assume that $x_{j} y_{i} \in I \backslash \mathfrak{p}$; since $\breve{I} \subseteq \mathfrak{p}$, $j \triangleright i$. First, we may also assume that for all $i^{\prime} \neq i, i \triangleright i^{\prime}$, if $x_{j} y_{i^{\prime}} \in I$, then $y_{i^{\prime}} \in \mathfrak{p}$, and similarly, that for all $j^{\prime} \neq j, j \triangleright j^{\prime}$, if $x_{j^{\prime}} y_{i} \in I$, then $x_{j^{\prime}} \in \mathfrak{p}$. Secondly, $i$ and $j$ belong to the same strong component of $\mathfrak{d}_{G}$; let $a$ be such that $i, j \in \mathcal{Z}_{a}$. Let $\mathcal{C}_{l}$ be chain of $P$ containing $x_{j} y_{i}$. For all $b \varsubsetneqq a$ and $j^{\prime} \in \mathcal{Z}_{b}, x_{j} y_{j^{\prime}} \in \breve{I} \subseteq \mathfrak{p}$, so $y_{j^{\prime}} \subseteq \mathfrak{p}$. Similarly, for all $b \supsetneqq a$ and $i^{\prime} \in \mathcal{Z}_{b}, x_{i^{\prime}} y_{i} \in \breve{I} \subseteq \mathfrak{p}$, so $x_{i^{\prime}} \subseteq \mathfrak{p}$. We can thus conclude that if $x_{j^{\prime}} y_{i^{\prime}} \in \mathcal{C}_{l}$ and $(i, j) \neq\left(i^{\prime}, j^{\prime}\right)$, then $x_{j^{\prime}} y_{i^{\prime}} \in \mathfrak{p}$. Therefore $x_{j} y_{i} \in \mathfrak{p}$, contradicting the choice of $x_{j} y_{i}$.

On $\mathbb{N}^{2}$, we define a poset by setting $(a, b) \geq(c, d)$ if $a \geq c$ and $b \geq d$. Let $(P, \geq)$, be a finite poset on a vertex set $W_{1}$. We say that $P$ can be embedded in $\mathbb{N}^{2}$ if there exists a map $\phi: W \longrightarrow \mathbb{N}^{2}$ such that all $i, j \in W, j \geq i$ if and only if $\phi(j) \geq \phi(i)$; such a map $\phi$ will be called an embedding of $P$ in $\mathbb{N}^{2}$. We will denote the projection of $\mathbb{N}^{2}$ along the first co-ordinate by $\pi$.

Definition 4.3 Let $(P, \succcurlyeq)$ be a finite poset on a finite vertex set $W$, with an embedding $\phi$ in $\mathbb{N}^{2}$. Then there is a unique $i_{0} \in W$ such that $i_{0}$ is minimal in $P$ and $(\pi \circ \phi)\left(i_{0}\right)$ is minimum. Similarly, let $j_{0}$ be the unique maximal element such that $(\pi \circ \phi)\left(j_{0}\right)$ is minimum. Let $P_{1}$ and $P_{2}$ be the restrictions of $P$ respectively to $W \backslash\left\{i_{0}\right\}$ and $W \backslash\left\{j_{0}\right\}$. The column linearization of $P$ induced by $\phi$ is the map $\gamma: W \longrightarrow[|W|]$ defined recursively as follows:

$$
\gamma(i)= \begin{cases}1, & i=i_{0} \\ 1+\gamma_{1}(i), & i \neq i_{0}\end{cases}
$$

where $\gamma_{1}$ is a column linearization of $P_{1}$ induced by $\phi$. A row linearization of $P$ induced by $\phi$ is the map $\rho: W \longrightarrow[|W|]$ defined recursively as follows:

$$
\rho(j)= \begin{cases}1, & j=j_{0} \\ 1+\rho_{1}(j), & j \neq j_{0}\end{cases}
$$

where $\rho_{1}$ is a row linearization of $P_{2}$ induced by $\phi$. We will say that $(\gamma, \rho)$ is the pair of linearizations induced by $\phi$.

Proposition 4.4 Let $P, \phi, \gamma$ and $\rho$ be as in Definition 4.3. For $i, j \in P$, if $j \succcurlyeq i$, $j \neq i$, then $\gamma(j)>\gamma(i)$ and $\rho(j)<\rho(i)$. If $i$ and $j$ are incomparable, then $\gamma(j)>$ $\gamma(i)$ if and only if $\rho(j)>\rho(i)$.

Proof If $j \succcurlyeq i$, then $\phi(j) \geq \phi(i)$. In the recursive definition of $\gamma, i$ would appear as the unique minimal vertex with the smallest value of $(\pi \circ \phi)$ before $j$ would, so 
$\gamma(i)<\gamma(j)$. On the other hand, while computing $\rho$ recursively, $j$ would appear as the unique maximal vertex with the smallest value of $(\pi \circ \phi)$ before $i$ would, so $\rho(j)<\rho(i)$. On the other hand, if $i$ and $j$ are incomparable, then we may assume without loss of generality that $(\pi \circ \phi)(i)<(\pi \circ \phi)(j)$. Hence, while computing $\gamma$ and $\rho$ recursively, $i$ will be chosen before $j$, giving $\gamma(i)<\gamma(j)$ and $\rho(i)<\rho(j)$.

Discussion 4.5 Let $P$ be a poset on a finite set $W$, with an embedding $\phi$ in $\mathbb{N}^{2}$. Let $(\gamma, \rho)$ be the pair of linearizations of $P$ induced by $\phi$. Let $E=\{(\gamma(i), \rho(j)): j \succcurlyeq$ $i \in W\} \subseteq \mathbb{R}^{2}$. We think of $E$ as a subset of $[|W|] \times[|W|]$ in the first quadrant of the Cartesian plane. Let $i, j$ be such that $(\gamma(i), \rho(j)) \in E$ is not the lowest vertex in its column, i.e., there exists $l$ such that $(\gamma(i), \rho(l))$ lies below $(\gamma(i), \rho(j))$. Then $j \succcurlyeq i, l \succcurlyeq i$ and, from Proposition 4.4, $l \neq i$. Therefore, again from Proposition 4.4, $\gamma(l)>\gamma(i)$ and $(\gamma(i), \rho(l))$ is not the right-most vertex in its row. Let $k$ be such that $(\gamma(k), \rho(l))$ lies immediately to the right of $(\gamma(i), \rho(l))$ in its row. Draw an edge between $(\gamma(i), \rho(j))$ and $(\gamma(k), \rho(l))$. Repeating this for all $j \succcurlyeq i$ such that $(\gamma(i), \rho(j))$ is not the lowest vertex in its column, we obtain a graph $\Gamma$ on $E$. Rows and columns of $\Gamma$ will be indexed starting from the bottom left corner.

Lemma 4.6 With notation as in Discussion 4.5, $\Gamma$ has exactly $|W|$ connected components.

Proof Suppose that $C$ is a connected component of $\Gamma$ and that $(\gamma(i), \rho(j))$ is the top left vertex of $C$. We claim that it is the left-most vertex in its row. For, if not, then there exists $k$ such that $(\gamma(k), \rho(j))$ lies immediately to the left of $(\gamma(i), \rho(j))$. From Proposition 4.4, $k \neq j$. We note, again from Proposition 4.4, that $(\gamma(k), \rho(j))$ is not the top-most vertex in its column, contradicting the hypothesis that $(\gamma(i), \rho(j))$ is the top left vertex of $C$. Now, there are exactly $|W|$ rows in $\Gamma$.

Lemma 4.7 Let $G$ be a Cohen-Macaulay bipartite graph such that $\phi$ is an embedding of $\mathfrak{d}_{G}$ in $\mathbb{N}^{2}$. Let $(\gamma, \rho)$ be the pair of linearizations induced by $\phi$. Then the vertices in the first column of $\Gamma$ belong to a contiguous set of rows, starting with row 1 .

Proof We may assume that the labelling of $\mathfrak{d}_{G}$ is such that $\gamma^{-1}(1)=1$ and $\gamma^{-1}(2)=2$. We need to show that $\rho(i)>\rho(1)$ if $i \neq 1$. Proposition 4.4 gives that 1 is minimal in $\mathfrak{d}_{G}$. Let $i \neq 1$. Then $i$ and 1 are incomparable. Since $\gamma(1)=1 \leq \gamma(i)$, we see, again from Proposition 4.4, that $\rho(i)>\rho(1)$.

Remark 4.8 Let $P$ be a poset on a finite vertex set $W$ with an embedding $\phi$ in $\mathbb{N}^{2}$. Let $(\gamma, \rho)$ be the pair of linearizations of $P$ induced by $\phi$. Let $W^{\prime}=W \backslash\left\{\gamma^{-1}(1)\right\}$ and let $P^{\prime}$ be the restriction of $P$ to $W^{\prime}$. Then $\left.\phi\right|_{W^{\prime}}$ is an embedding of $P^{\prime}$ in $\mathbb{N}^{2}$. For $i \in W^{\prime}$, set $\gamma^{\prime}(i)=\gamma(i)-1$, and

$$
\rho^{\prime}(i)= \begin{cases}\rho(i), & i \succcurlyeq \gamma^{-1}(1) \\ \rho(i)-1, & \text { otherwise. }\end{cases}
$$


Then $\left(\gamma^{\prime}, \rho^{\prime}\right)$ is the pair of linearizations induced by $\left.\phi\right|_{W^{\prime}}$. Let $\Gamma^{\prime}$ be the graph constructed from $P^{\prime}$ as described in Discussion 4.5 using $\gamma^{\prime}$ and $\rho^{\prime}$. Then $\Gamma^{\prime}$ is obtained by deleting the vertices in the first column of $\Gamma$. We see this as follows. For all $i, j \in W^{\prime}, \rho(i)<\rho(j)$ if and only if $\rho^{\prime}(i)<\rho^{\prime}(j)$; similarly, $\gamma(i)<\gamma(j)$ if and only if $\gamma^{\prime}(i)<\gamma^{\prime}(j)$. Further, there is only one vertex in row $\rho\left(\gamma^{-1}(1)\right)$ in $\Gamma$, and this is in the first column.

Remark 4.9 Let $P$ be a poset on a finite vertex set $W$ with an embedding $\phi$ in $\mathbb{N}^{2}$. Let $(\gamma, \rho)$ be the pair of linearizations induced by $\phi$. Let $W^{\prime}=W \backslash \gamma^{-1}(1)$ and let $P^{\prime}$ be the restriction of $P$ to $W^{\prime}$. Then $\left.\phi\right|_{W^{\prime}}$ is an embedding of $P$ in $\mathbb{N}^{2}$. Let $\tilde{\gamma}$ be the order-preserving map from $\left.\operatorname{Im} \gamma\right|_{W^{\prime}}$ to $\left[\left|W^{\prime}\right|\right]$. Let $\gamma^{\prime}:=\left.\tilde{\gamma} \circ \gamma\right|_{W^{\prime}}$. For $j \in W^{\prime}$, set $\rho^{\prime}(j)=\rho(j)-\rho(1)$. Then $\left(\gamma^{\prime}, \rho^{\prime}\right)$ is the pair of linearizations of $P^{\prime}$ induced by $\left.\phi\right|_{W^{\prime}}$. Let $\Gamma^{\prime}$ be the graph constructed from $P^{\prime}$ as described in Discussion 4.5 using $\left.\tilde{\gamma} \circ \gamma\right|_{W^{\prime}}$ and $\left.\tilde{\rho} \circ \rho\right|_{W^{\prime}}$. We claim that $\Gamma^{\prime}$ is the graph obtained from $\Gamma$ by deleting the vertices that lie in rows $\rho(j)$ for some $j \succcurlyeq \gamma^{-1}(1)$. For, first observe that for all $i, j \in W^{\prime}, \rho(i)<\rho(j)$ if and only if $\rho^{\prime}(i)<\rho^{\prime}(j)$; similarly, $\gamma(i)<\gamma(j)$ if and only if $\gamma^{\prime}(i)<\gamma^{\prime}(j)$. Moreover, for all $j \succcurlyeq \gamma^{-1}(1)$, the vertices in the column $\gamma(j)$ belong to rows between 1 and $\rho(j)$ (possibly, not all of them). Therefore, after the vertices in the rows between 1 and $\rho(1)$ have been deleted, the remaining vertices belong to columns $\gamma(j)$ for $j \neq 1$. Hence $\left(\gamma^{\prime}(i), \rho^{\prime}(j)\right)$ and $\left(\gamma^{\prime}(k), \rho^{\prime}(l)\right)$ belong to the same connected component of $\Gamma^{\prime}$ if and only if $(\gamma(i), \rho(j))$ and $(\gamma(k), \rho(l))$ belong to the same connected component of $\Gamma$.

Example 4.10 We wish to illustrate these constructions with an example of a CohenMacaulay bipartite graph. Let $G$ be the Cohen-Macaulay bipartite graph on the vertex set $\left\{x_{1}, y_{1}, \ldots, x_{7}, y_{7}\right\}$ such that the poset $\mathfrak{d}_{G}$ has the cover relations (i.e., chains that cannot be further refined) $3 \succ 1,3 \succ 2,4 \succ 1,4 \succ 2,5 \succ 2,6 \succ 3,6 \succ 4,7 \succ 4$ and $7 \succ 5$. Table 1 gives the embedding $\phi$, the functions $\gamma$ and $\rho$ and the graph $\Gamma$. We take the sum of the monomials corresponding to the vertices in a connected component of $\Gamma$ :

$$
\begin{array}{ll}
g_{1}=x_{1} y_{6}, \quad g_{2}=x_{2} y_{6}+x_{1} y_{3}, & g_{3}=x_{3} y_{6}+x_{2} y_{3}+x_{1} y_{7}, \\
g_{4}=x_{4} y_{6}+x_{3} y_{3}+x_{2} y_{7}+x_{1} y_{4}, & g_{5}=x_{6} y_{6}+x_{4} y_{7}+x_{2} y_{4}+x_{1} y_{1}, \\
g_{6}=x_{5} y_{7}+x_{4} y_{4}+x_{2} y_{5}, & g_{7}=x_{7} y_{7}+x_{5} y_{5}+x_{2} y_{2} .
\end{array}
$$

Let $J=\left(g_{1}, \ldots, g_{7}\right)$. In the proof of Proposition 4.11 we will see that $I=\sqrt{J}$.

Before we prove the second assertion of Theorem 1.3, we observe that the directed graph associated to $\breve{G}$ (which we denoted by $\breve{\mathfrak{d}}$ in Discussion 4.1 ) has an embedding in $\mathbb{N}^{2}$ if and only if the acyclic reduction $\widehat{\mathfrak{d}}$ of $\mathfrak{d}_{G}$ has an embedding in $\mathbb{N}^{2}$. The proof of this is easy, and is omitted.

Proposition 4.11 Let $G$ be an unmixed bipartite graph. If a maximal transitively closed and acyclic subgraph of $\mathfrak{d}_{G}$ can be embedded in $\mathbb{N}^{2}$, then ara $I=\operatorname{pd} R / I$. 
Table 1 Example 4.10

\begin{tabular}{|c|ccc|}
\hline$i$ & $\phi(i)$ & $\gamma(i)$ & $\rho(i)$ \\
\hline 1 & $(0,2)$ & 1 & 5 \\
2 & $(1,0)$ & 2 & 7 \\
3 & $(2,5)$ & 3 & 2 \\
4 & $(3,3)$ & 4 & 4 \\
5 & $(5,1)$ & 6 & 6 \\
6 & $(4,6)$ & 5 & 1 \\
7 & $(6,4)$ & 7 & 3 \\
\hline
\end{tabular}

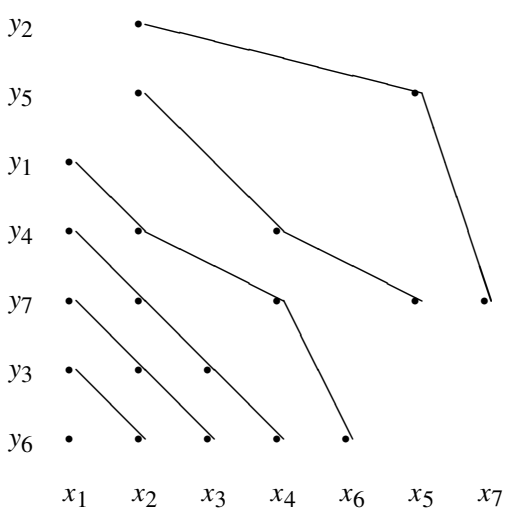

Proof Let $\breve{\mathfrak{d}}$ be a maximal acyclic subgraph of $\mathfrak{d}_{G}$ with the property that $\breve{\mathfrak{d}}$ can be embedded in $\mathbb{N}^{2}$. Construct $\breve{G}$ as in Discussion 4.1. Let $\breve{I}$ be its edge ideal. Observe that $\breve{G}$ is Cohen-Macaulay and ht $\breve{I}=\mathrm{ht} I=c$. Suppose that the conclusion of the proposition holds for Cohen-Macaulay graphs. Then ara $\breve{I}=\operatorname{pd} R / \breve{I}=\mathrm{ht} I$. Using Proposition 4.2 and the fact that ara $I \geq \operatorname{pd} R / I$ ([8, Proposition 3]), we conclude that ara $I=\operatorname{pd} R / I$. Hence it suffices to prove the assertion in the Cohen-Macaulay case. Assume, therefore, that $G$ is Cohen-Macaulay.

Denote the embedding of $\mathfrak{d}_{G}$ by $\phi$, and let $(\gamma, \rho)$ be pair of linearizations induced by $\phi$. Let $\Gamma$ be the graph constructed as in Discussion 4.5. We prove the theorem by induction on $c$. Since the conclusion is evident when $c=1$, we assume that $c>1$ and that it holds for all Cohen-Macaulay bipartite graphs on fewer than $2 c$ vertices. For $t=1, \ldots, c$, let $C_{t}$ be the connected component of $\Gamma$ containing the left most vertex in row $t$. We saw in the proof of Lemma 4.6 that these are exactly the connected components of $\Gamma$. Set

$$
g_{t}=\sum_{(\gamma(i), \rho(j)) \in C_{t}} x_{i} y_{j} \quad 1 \leq t \leq c
$$

Set $J=\left(g_{1}, \ldots, g_{c}\right)$. We will show that $I=\sqrt{J}$, or, equivalently, that for all $\mathfrak{p} \in$ Spec $R, I \subseteq \mathfrak{p}$ if and only if $J \subseteq \mathfrak{p}$. (This gives that ara $I \leq c=\operatorname{pd} R / I=$ ht $I$, but we have already noted that ara $I \geq=\operatorname{pd} R / I$.) Further, without loss of generality, we may assume that $\gamma^{-1}(1)=1$. Then 1 is a minimal element of $\mathfrak{d}_{G}$. Let $W_{1}:=\{2, \ldots, c\}$ and $W_{2}:=\{i \neq 1\} \subseteq[c]$. Let $\mathfrak{d}_{1}$ and $\mathfrak{d}_{2}$ respectively be the restrictions of $\mathfrak{d}_{G}$ to $W_{1}$ and $W_{2}$.

Let $G_{1}$ be the deletion of $x_{1}$ and $y_{1}$ in $G$, whose edge ideal (in $R=\mathbb{k}[V]$ ) is $\left(\left(I, x_{1}\right) \cap \mathbb{k}\left[x_{2}, y_{2}, \ldots, x_{c}, y_{c}\right]\right) R$. Note that $\mathfrak{d}_{1}$ is the associated directed graph of $G_{1}$. Let $\Gamma_{1}$ denote the deletion of the vertices that lie in the first column of $\Gamma$. Write $J_{1}=$ $\left(\left(J, x_{1}\right) \cap \mathbb{k}\left[x_{2}, y_{2}, \ldots, x_{c}, y_{c}\right]\right) R$. We see from Remark 4.8 that $J_{1}$ is defined from $\Gamma_{1}$ precisely the same way that $J$ is defined from $\Gamma$. Along with the induction hypothesis, this gives that $\left(\left(I, x_{1}\right) \cap \mathbb{k}\left[x_{2}, y_{2}, \ldots, x_{c}, y_{c}\right]\right) R=\sqrt{J_{1}}$. Note that $\left(J_{1}, x_{1}\right)=\left(J, x_{1}\right)$, so we obtain that $\left(I, x_{1}\right)=\sqrt{\left(J, x_{1}\right)}$. We thus see that for all $\mathfrak{p} \in \operatorname{Spec} R$ such that $x_{1} \in \mathfrak{p}, I \subseteq \mathfrak{p}$ if and only if $J \subseteq \mathfrak{p}$. 
Let $G_{2}$ be the deletion of $x_{1}$ and all its neighbours in $G$; its edge ideal is ( $I$ : $\left.\left.x_{1}\right) \cap \mathbb{k}\left[x_{i}, y_{i}: i \in W_{2}\right]\right) R$. The associated directed graph of $G_{2}$ is $\mathfrak{d}_{2}$. Let $\Gamma_{2}$ denote the deletion of the vertices that lie in columns $\gamma(i)$ or in rows $\rho(i)$ of $\Gamma$ whenever $i \succcurlyeq 1$. Let

$$
J_{2}=\left(\left(J+\left(y_{i}: i \succcurlyeq 1\right)\right) \cap \mathbb{k}\left[x_{i}, y_{i}: i \neq 1\right]\right) R .
$$

From Remark 4.8, we note that $J_{2}$ is defined from $\Gamma_{2}$ precisely the same way that $J$ is defined from $\Gamma$. This, along with the induction hypothesis, implies that $((I$ : $\left.\left.x_{1}\right) \cap \mathbb{k}\left[x_{i}, y_{i}: i \in W_{2}\right]\right) R=\sqrt{J_{2}}$. Now, $J_{2}+\left(y_{i}: i \succcurlyeq 1\right)=J+\left(y_{i}: i \succcurlyeq 1\right)=\left(J: x_{1}\right)$, so $\left(I: x_{1}\right)=\sqrt{\left(J: x_{1}\right)}$. We thus see that for all $\mathfrak{p} \in \operatorname{Spec} R$ such that $x_{1} \notin \mathfrak{p}, I \subseteq \mathfrak{p}$ if and only if $J \subseteq \mathfrak{p}$. Together with the previous paragraph, we conclude that $\sqrt{J}=I$.

Acknowledgements This work was done as part of the author's dissertation at the University of Kansas, under the direction of C. Huneke. He thanks Huneke and J. Martin for helpful discussions, and the referees for their comments. The computer algebra system Macaulay2 by D. Grayson and M. Stillman provided valuable assistance in studying examples.

\section{References}

1. Barile, M.: On the number of equations defining certain varieties. Manuscripta Math. 91(4), 483-494 (1996). MR MR1421287 (97m:13041)

2. Barile, M.: A note on monomial ideals. Arch. Math. (Basel) 87(6), 516-521 (2006). MR MR2283682 (2007h:13004)

3. Bruns, W., Herzog, J.: Cohen-Macaulay Rings. Cambridge Studies in Advanced Mathematics, vol. 39. Cambridge University Press, Cambridge (1993). MR 95h:13020

4. Herzog, J., Hibi, T.: Distributive lattices, bipartite graphs and Alexander duality. J. Algebr. Comb. 22(3), 289-302 (2005). MR MR2181367 (2006h:06004)

5. Hà, H.T., Van Tuyl, A.: Monomial ideals, edge ideals of hypergraphs, and their graded Betti numbers. J. Algebr. Comb. 27(2), 215-245 (2008). MR MR2375493 (2009a:05145)

6. Katzman, M.: Characteristic-independence of Betti numbers of graph ideals. J. Comb. Theory Ser. A 113(3), 435-454 (2006). MR MR2209703 (2007f:13032)

7. Kimura, K., Terai, N., Yoshida, K.-i.: Arithmetical rank of squarefree monomial ideals of small arithmetic degree, J. Algebr. Comb. (to appear)

8. Lyubeznik, G.: On the arithmetical rank of monomial ideals. J. Algebra 112(1), 86-89 (1988). MR MR921965 (89b:13020)

9. Miller, E., Sturmfels, B.: Combinatorial Commutative Algebra. Graduate Texts in Mathematics, vol. 227. Springer-Verlag, New York (2005). MR MR2110098 (2006d:13001)

10. Stanley, R.P.: Enumerative Combinatorics. Vol. 1. Cambridge Studies in Advanced Mathematics, vol. 49. Cambridge University Press, Cambridge (1997). With a foreword by Gian-Carlo Rota, Corrected reprint of the 1986 original. MR MR1442260 (98a:05001)

11. Schenzel, P., Vogel, W.: On set-theoretic intersections. J. Algebra 48(2), 401-408 (1977). MR MR0472852 (57 \#12541)

12. Schmitt, T., Vogel, W.: Note on set-theoretic intersections of subvarieties of projective space. Math. Ann. 245(3), 247-253 (1979). MR MR553343 (81a:14025)

13. Terai, N.: Alexander duality theorem and Stanley-Reisner rings. Sūrikaisekikenkyūsho Kōkyūroku 1078, 174-184 (1999). Free resolutions of coordinate rings of projective varieties and related topics (in Japanese) (Kyoto, 1998). MR MR1715588 (2001f:13033)

14. Villarreal, R.H.: Monomial Algebras. Monographs and Textbooks in Pure and Applied Mathematics, vol. 238. Marcel Dekker, New York (2001). MR MR1800904 (2002c:13001)

15. Villarreal, R.H.: Unmixed bipartite graphs. Rev. Colombiana Mat. 41(2), 393-395 (2007)

16. West, D.B.: Introduction to Graph Theory. Prentice-Hall, Upper Saddle River (1996). MR MR1367739 (96i:05001) 
17. Yan, Z.: An étale analog of the Goresky-MacPherson formula for subspace arrangements. J. Pure Appl. Algebra 146(3), 305-318 (2000). MR MR1742346 (2000k:14041)

18. Yanagawa, K.: Alexander duality for Stanley-Reisner rings and squarefree $\mathbb{N}^{n}$-graded modules. J. Algebra 225(2), 630-645 (2000). MR MR1741555 (2000m:13036)

19. Zheng, X.: Resolutions of facet ideals. Commun. Algebra 32(6), 2301-2324 (2004). MR MR2100472 (2006c:13034) 